Real Analysis Exchange

Vol. 24(2), 1998/9, pp. 821-826

T. H. Steele, Department of Mathematics, Weber State University, Ogden, UT, 84408-1702; e-mail: thsteele@weber.edu

\title{
THE SET OF CONTINUOUS FUNCTIONS WITH ZERO TOPOLOGICAL ENTROPY
}

\begin{abstract}
Let $I=[0,1]$. We show that those functions in $C(I, I)$ possessing zero topological entropy form a nowhere dense perfect subset of the continuous self maps of the interval. We also show that every function with zero topological entropy that possesses an infinite $\omega$-limit set is the uniform limit of functions having only finite $\omega$-limit sets.
\end{abstract}

\section{Introduction}

In the study of chaotic and dynamical systems, those functions possessing zero topological entropy have received considerable attention. Topological entropy, as introduced in [1], was initially used to provide a numerical measure for the complexity of an endomorphism of a compact topological space. The notion has since been extended to provide a way of describing the chaotic behavior of a self map of a compact interval. As the following results of Misiurewicz and Sarkovskii indicate, those functions with zero topological entropy must have relatively benign iterative structures [8], [9], [7].

Theorem 1. Let $f:[0,1] \rightarrow[0,1]$ be continuous. The following conditions are equivalent.

- $f$ has positive topological entropy.

- For some $x \in I=[0,1]$, the $\omega$-limit set $\omega(x, f)$ is infinite and contains a periodic point.

- $f$ has a cycle of order not a power of two.

- There are closed intervals $J$ and $K$ in $I$ having at most one point in common, and positive integers $m, n$ such that $J \cup K \subset f^{m}(J) \cap f^{n}(K)$.

Key Words: topological entropy, continuous function, $\omega$-limit set

Mathematical Reviews subject classification: 54H20, 26A18

Received by the editors May 22, 1998 
Four years later V. Fedorenko, A. Sarkovskii and J. Smítal published a more extensive list of conditions equivalent to a function having zero topological entropy; the interested reader is referred to Theorem A of [6]. From Theorem 1, one sees that each periodic orbit of $f$ must have order $2^{n}$ for some nonnegative integer $n$ whenever $f$ has zero topological entropy. Our next theorem, due to Smítal, sheds considerable light onto the structure of the infinite $\omega$-limit sets of a function with zero topological entropy [10].

Theorem 2. If $\omega$ is an infinite $\omega$-limit set of $f \in C(I, I)$ possessing zero topological entropy, then there exists a sequence of closed intervals $\left\{J_{k}\right\}_{k=1}^{\infty}$ in $[0,1]$ such that

- for each $k,\left\{f^{i}\left(J_{k}\right)\right\}_{i=1}^{2^{k}}$ are pairwise disjoint, and $J_{k}=f^{2^{k}}\left(J_{k}\right)$.

- for each $k, J_{k+1} \cup f^{2^{k}}\left(J_{k+1}\right) \subset J_{k}$.

- for each $k, \omega \subset \cup_{i=1}^{2^{k}} f^{i}\left(J_{k}\right)$.

- for each $k$ and $i, \omega \cap f^{i}\left(J_{k}\right) \neq \emptyset$.

Given the very specific behavior that functions of zero topological entropy must demonstrate on their infinite $\omega$-limit sets, it may not be too surprising that Bruckner and Smital have been able to characterize these sets [4].

Theorem 3. An infinite compact set $W \subset(0,1)$ is an $\omega$-limit set of a map $f \in C(I, I)$ with zero topological entropy if and only if $W=Q \cup P$ where $Q$ is a Cantor set and $P$ is empty or countably infinite, disjoint with $Q$, and satisfies the following conditions:

- every interval contiguous to $Q$ contains at most two points of $P$;

- each of the intervals $[0, \min Q),(\max Q, 1]$ contains at most one point of $P$, and

- $\bar{P}=Q \cup P$.

More recently in [2], Block and Coppel have turned their attention to the structure of the collection $\mathcal{E}=\{f \in C(I, I): f$ has zero topological entropy $\}$ as a subset of $C(I, I)$. We extend their results by first recalling that $\mathcal{E}$ is a nowhere dense perfect subset of $C(I, I)$. We then go on to prove that every function in $\mathcal{E}$ possessing an infinite $\omega$-limit set is the uniform limit of functions possessing only finite $\omega$-limit sets. That $\mathcal{E}$ is a nowhere dense perfect subset of $C(I, I)$ follows from earlier work of Block and Misiurewicz; we present an alternate proof of this fact since it complements the second part of Theorem 4, which answers a specific query of [2]. 


\section{Preliminaries}

We write $\mathbf{h}(f)=0$ in order to indicate that $f$ in $C(I, I)$ possesses zero topological entropy, so that $\mathcal{E}=\{f \in C(I, I): \mathbf{h}(f)=0\}$. While we can take $I$ to be any compact interval of the real line, for convenience we set $I=[0,1]$. We let $\|f-g\|=\sup \{|f(x)-g(x)|: x \in I\}$, and work in the complete metric space $(C(I, I),\|\circ\|)$. Also, let $\mathbb{P}_{n}$ denote the set of all continuous functions $f: I \rightarrow I$ which possess a point of period $n$, and let $\mathbb{P}_{2 \infty}$ represent those functions with zero topological entropy that possess a point of period $2^{n}$ for all natural numbers $n$. From the Sarkovskii ordering on periodic orbits of continuous self-maps of a compact interval, one sees that $\mathbb{P}_{2^{\infty}} \subset \cdots \subset \mathbb{P}_{2^{n+1}} \subset \mathbb{P}_{2^{n}} \subset \cdots \subset \mathbb{P}_{4} \subset \mathbb{P}_{2} \subset \mathbb{P}_{1}$

We make the following definitions with Smítal's Theorem 2 in mind. Let $\omega$ be an infinite compact subset of $I$, and let $f$ map $\omega$ into itself. We call $f$ a simple map on $\omega$ if $\omega$ has a decomposition $S \cup T$ into compact portions that $f$ exchanges, and $f^{2}$ is simple on each of these portions. From Smítal's Theorem one sees that every map $f$ with zero topological entropy is simple on each of its infinite $\omega$-limit sets. Let $\left\{J_{k}\right\}_{k=1}^{\infty}$ be a nested sequence of compact periodic intervals of $\omega$ and $f$ as described in Smítal's Theorem. Every set of the form $\omega \cap f^{i}\left(J_{k}\right)$ is periodic of period $2^{k}$, and we call each such set a periodic portion of rank $k$. This system of periodic portions of $\omega$, or of the corresponding periodic intervals, is called the simple system of $\omega$ with respect to $f$. We now recall a device from [5] that allows us to code the sets $f^{i}\left(J_{k}\right)$ with finite tuples of zeros and ones. Let $\mathbb{N}$ denote the natural numbers, and take $\mathcal{N}$ to be the set of sequences composed of zeros and ones. If $\mathbf{n} \in \mathcal{N}$ and $\mathbf{n}=\left\{n_{i}\right\}_{i=1}^{\infty}$, we let $\mathbf{n} \mid k=\left(n_{1}, n_{2}, \ldots, n_{k}\right)$. Set $\mathbf{0}=\{0,0, \ldots\}$ and $\mathbf{1}=\{1,1, \ldots\}$. Now, define a function $\mathcal{A}: \mathcal{N} \longrightarrow \mathcal{N}$ given by $\mathcal{A}(\mathbf{n})=\mathbf{n}+10$, where addition is modulus two from left to right. For each $k \in \mathbb{N}$ and $i \in \mathbb{N}$ put $F_{\mathbf{1} \mid k}=J_{k}$ and $F_{\mathcal{A}^{i}(\mathbf{1} \mid k)}=f^{i}\left(J_{k}\right)$. Thus, for each $\mathbf{m}$ and $\mathbf{n}$ in $\mathcal{N}$ and $k \in \mathbb{N}$ there is a $j \in \mathbb{N}$ such that $\mathcal{A}^{j}(\mathbf{m} \mid k)=\mathbf{n} \mid k$; the above relations define $F_{\mathbf{n} \mid k}$ for all $\mathbf{n} \in \mathcal{N}$ and $k \in \mathbb{N}$. Now, set $F_{\mathbf{n}}=\cap_{k=1}^{\infty} F_{\mathbf{n} \mid k}$, and let $K=\cup_{\mathbf{n} \in \mathcal{N}} \cap_{k=1}^{\infty} F_{\mathbf{n} \mid k}$. Then $K$ and each $F_{\mathbf{n}}$ are compact, and the components of $K$ consist of the $F_{\mathbf{n}}$ sets. Let $G$ be the component of $[0,1]-K$ which contains the interval between $F_{1}$ and $F_{0}$. In general, let $G_{\mathbf{n} \mid k}$ be that component of $[0,1]-K$ which contains the interval between $F_{\mathbf{n} \mid k 0}$ and $F_{\mathbf{n} \mid k 1}$. We set $\mathcal{G}=\{G\} \cup\left\{G_{\mathbf{n} \mid k}: \mathbf{n}\right.$ $\in \mathcal{N}, k \in \mathbb{N}\}, G^{0}=G \cup[0, \inf K) \cup(\sup K, 1]$ and $G^{k}=\cup\left\{G_{\mathbf{n} \mid k}: \mathbf{n} \in \mathcal{N}\right\}$. Thus, $[\inf K, \sup K]=K \cup \mathcal{G}$ and $[0,1]=K \cup\left(\cup_{j=0}^{\infty} G^{j}\right)$. 


\section{$3 \quad$ Results}

We are now in a position to state precisely as well as prove our main result; this is the content of the following theorem.

Theorem 4. Let $\mathcal{E}=\{f \in C(I, I): \mathbf{h}(f)=0\}$. Then

- $\mathcal{E}$ is a nowhere dense perfect subset of $C(I, I)$.

- $\overline{\cup_{k \geq 0}\left(\mathbb{P}_{2^{k}} \cap \mathcal{E}\right)}=\mathcal{E}$.

Proof. We first prove that $\mathcal{E}$ is a nowhere dense closed subset of $C(I, I)$. From [2] we know that the function $\mathbf{h}: C(I, I) \rightarrow \mathbb{R}^{+} \cup\{+\infty\}$, given by $f \rightarrow \mathbf{h}(f)$, is lower semicontinuous. Thus, if $\mathbf{h}(f)>\alpha>0$, then $\mathbf{h}(g)>\alpha$ for all $g \in C(I, I)$ sufficiently close to $f$. In particular, the set $\mathcal{S}=\{f \in$ $C(I, I): \mathbf{h}(f)>0\}$ is open. Now, suppose that $f \in \mathcal{E}$. Since $f$ must have a fixed point in $I$, for any $\varepsilon>0$ we can find $g$ in $C(I, I)$ so that $\|f-g\|<\varepsilon$, yet $\mathbf{h}(g)>0$. In fact, we can take $g$ to equal $f$ outside of a neighborhood of the fixed point, and define $g$ on a subinterval of that neighborhood so that it is an appropriately scaled copy of the hat map $h: I \rightarrow I$ given by $x \longmapsto 2 x$ for $x \in\left[0, \frac{1}{2}\right]$, and $x \longmapsto 2(1-x)$ for $x \in\left[\frac{1}{2}, 1\right]$. To show that $\mathcal{E}$ is perfect in addition to being nowhere dense and closed in $C(I, I)$, we let $\left\{\varphi_{n}\right\}$ be a sequence of homeomorphisms from $I$ to $I$ that converge uniformly to the identity map on $I$. Let $f$ be an element of $\mathcal{E}$, and set $f_{n}=\varphi_{n} f \varphi_{n}^{-1}$. Since $f_{n}$ is topologically conjugate to $f \in \mathcal{E}$, one sees that $\mathbf{h}\left(f_{n}\right)=0$ for each $n$, too. Moreover, $f_{n} \rightarrow f$ since the uniform limit of $\left\{\varphi_{n}\right\}$ is the identity map.

It remains to show that $\overline{\cup_{k>0}\left(\mathbb{P}_{2^{k}} \cap \mathcal{E}\right)}=\mathcal{E}$. To this end, it suffices to show that for any $f$ in $\mathbb{P}_{2^{\infty}}$, there exists $\left\{f_{n}\right\}$ contained in $\cup_{k>0}\left(\mathbb{P}_{2^{k}} \cap \mathcal{E}\right)$ for which $f_{n} \rightarrow f$. Let $f \in \mathbb{P}_{2^{\infty}}$. We proceed through two cases. First, let us suppose that $f$ is not chaotic in the sense of $\mathrm{Li}$ and Yorke, so that $\left|F_{\mathbf{n}}\right|=0$ for all the components $F_{\mathbf{n}}$ in a simple system of $f$, should the function possess an infinite $\omega$-limit set [3]. Let $\varepsilon>0$. Then there exists $N \in \mathbb{N}$ with the property that $\left|F_{\mathbf{n} \mid k}\right|<\varepsilon$ for all $\mathbf{n}$ in $\mathcal{N}$, whenever $k \geq N$. We let $g$ equal $f$ on $I-\left(\cup_{\mathbf{n} \in \mathcal{N}} F_{\mathbf{n} \mid N}\right)$, and extend $g$ linearly on each of the $2^{N}$ intervals of the form $F_{\mathbf{n} \mid N}$. Since $g^{2^{N}}: F_{\mathbf{n} \mid N} \rightarrow F_{\mathbf{n} \mid N}$ is linear for each $\mathbf{n}$ in $\mathcal{N}$, it follows that $g$ has periodic points of order no more that $2^{N+1}$. In a similar fashion one constructs $g$ a $2^{N+1}$ function so that $\|f-g\|<\varepsilon$ whenever $f$ is a $2^{\infty}$ function that does not possess an infinite $\omega$-limit set.

For our second case, let us suppose that $f$ is chaotic in the sense of $\mathrm{Li}$ and Yorke, so that $\operatorname{int} F_{\mathbf{n}} \neq \emptyset$ for some component of the simple system of $f$. Let $x \in \operatorname{int} F_{\mathbf{n}}$, and take $\varepsilon>0$. Since $f$ is uniformly continuous, there exists $N$ a natural number such that $\left|G_{\mathbf{m} \mid n}\right|<\varepsilon$ and $\left|f\left(G_{\mathbf{m} \mid n}\right)\right|<\varepsilon$ whenever $n>N$ 
for all $\mathbf{m}$ in $\mathcal{N}$. Also, there exists $\mathbf{m}$ in $\mathcal{N}$ and $M$ in $\mathbb{N}$ so that $\left|F_{\mathbf{m} \mid n}\right|<\varepsilon$ and $\left|F_{\mathcal{A}(\mathbf{m}) \mid n}\right|<\varepsilon$ for any $n>M$. Let $n>\max \{M, N\}$, and without loss of generality we may presume that $F_{\mathbf{m} \mid n+2}$ lies between $G_{\mathbf{m} \mid n}$ and $G_{\mathbf{m} \mid n+1}$. There exists a $k \in \mathbb{N}$ for which $f^{k}(x) \in F_{\mathbf{m} \mid n+2}$, and since $f^{2^{n+2}-k}\left(F_{\mathbf{m} \mid n+2}\right)=$ $F_{\mathbf{n} \mid n+2}$, there is $x^{*}$ in $F_{\mathbf{m} \mid n+2}$ such that $f^{2^{n+2}-k}\left(x^{*}\right)=x$. We modify $f$ on $F_{\mathbf{m} \mid n+2}, G^{n}$ and $G^{n+1}$ to get a function $g$ so that $g \in \mathbb{P}_{2^{n+2}}$, and $\|f-g\|<\varepsilon$. If $y$ is in $I-\left(F_{\mathbf{m} \mid n+2} \cup G^{n} \cup G^{n+1}\right)$, we let $g(y)=f(y)$. If $y \in F_{\mathbf{m} \mid n+2}$, let $g(y)=f\left(x^{*}\right)$. We extend $g$ in a linear fashion on $G^{n}$ and $G^{n+1}$ to get $g \in C(I, I)$ for which $\|f-g\|<\varepsilon$. Since $g^{k}(x)=f^{k}(x) \in F_{\mathbf{m} \mid n+2}, g\left(g^{k}(x)\right)=$ $f\left(x^{*}\right)$ and $g^{2^{n+2}-(k+1)}\left(g\left(g^{k}(x)\right)\right)=f^{2^{n+2}-(k+1)}\left(f\left(x^{*}\right)\right)=x$, it follows that $x$ has period $2^{n+2}$. Since $g$ possesses a unique periodic point of period $2^{n}$ in $G^{n}$, and a unique periodic point of period $2^{n+1}$ in $G^{n+1}$, it follows that $g$ is a $2^{n+2}$ function.

\section{References}

[1] R. Adler, A. Konheim and M. McAndrew, Topological entropy, Trans. Amer. Math. Soc. 114 (1965), 309-319.

[2] L. Block and W. Coppel, Dynamics in one dimension, Lecture Notes in Mathematics, vol. 1513, Springer-Verlag, 1991.

[3] A. M. Bruckner and J. G. Ceder, Chaos in terms of the map $x \longmapsto \omega(x, f)$, Pac. J. Math. 156 (1992), 63-96.

[4] A. M. Bruckner and J. Smítal, The structure of $\omega$-limit sets of maps of the interval with zero topological entropy, Ergod. Th. and Dynam. Sys. 13 (1993), 7-19.

[5] R. L. Devaney, Chaotic dynamical systems, Benjamin/Cummings Publ. Co., 1986.

[6] V. Fedorenko, A. Sarkovskii and J. Smítal, Characterizations of weakly chaotic maps of the interval, Proc. Amer. Math. Soc. 110 (1990), $141-148$.

[7] M. Misiurewicz, Horseshoe mappings of the interval, Bull. Acad. Polon. Sci. Ser. Math. 27 (1979), 167-169.

[8] A. N. Sarkovskii, The behavior of a map in a neighborhood of an attracting set, Ukrain. Mat Z. 18 (1966), 60-83. (in Russian) 
[9] A. N. Sarkovskii, On cycles and the structure of continuous mappings, Ukrain. Mat Z. 17 (1965), 104-111. (in Russian)

[10] J. Smítal, Chaotic functions with zero topological entropy, Trans. Amer. Math. Soc. 297 (1986), 269-282. 